Authors have nothing to disclose with regard to commercial support.

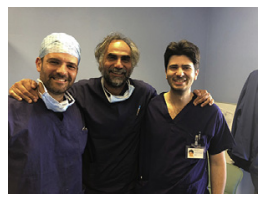

\section{CONVENTIONAL OR OXYGEN \\ DELIVERY-GUIDED \\ PERFUSION: WHICH \\ COMES FIRST, THE \\ CHICKEN OR \\ THE EGG?}

\section{To the Editor:}

Postoperative acute kidney injury (AKI) remains one of the most serious complications of cardiac surgery due to its frequency and association with mortality. Among the causative factors, cardiopulmonary bypass (CPB), hemodilution, and the need for maintaining oxygen delivery $\left(\mathrm{DO}_{2}\right)$ on CPB above the critical value have been studied extensively. In this regard, we read with great interest the multicenter trial of Ranucci and colleagues, ${ }^{1}$ who compared patients treated with a goaldirected perfusion strategy versus patients treated with a conventional perfusion approach targeted on body surface area and CPB temperature.

Although this issue is difficult to discriminate because of the high interdependence of several factors, we agree with the authors regarding the effectiveness of $\mathrm{DO}_{2}$-guided perfusion, but some methodologic aspects of the trial may have led to underestimating its potential advantages.

First, the duration of CPB observed in study patients was less than anticipated when designing the study $(27.6 \%$ vs $14 \%$ in the study arm), and this may have contributed to reduced frequency of the primary end-point. Second, assessment of serum creatinine levels limited to the first 48 hours after surgery may be too early to detect any significant changes, as confirmed by the fact that serum creatinine increase did not differ between groups. Measurement of other rapid markers of AKI (eg, neutrophil gelatinaseassociated lipocalin) could better provide evidence of the effectiveness of treatment. ${ }^{2}$

Third, in our opinion, the subgroup analysis including patients with CPB time between 1 and 3 hours should be

The Editor welcomes submissions for possible publication in the Letters to the Editor section that consist of commentary on an article published in the Journal or other relevant issues. Authors should: • Include no more than 500 words of text, three authors, and five references. $\bullet$ Type with double-spacing. $\bullet$ See http://jtcs.ctsnetjournals.org/ misc/ifora.shtml for detailed submission instructions. • Submit the letter electronically via jtcvs.editorialmanager.com. Letters commenting on an article published in the JTCVS will be considered if they are received within 6 weeks of the time the article was published. Authors of the article being commented on will be given an opportunity of offer a timely response ( 2 weeks) to the letter. Authors of letters will be notified that the letter has been received. Unpublished letters cannot be returned. further subdivided: there are substantial differences in the risk for AKI between patients undergoing surgery with a short CPB time and those undergoing complex operations with a CPB time of 3 hours. The authors themselves know well how a minute can make the difference in terms of final outcome. ${ }^{3}$ It would be interesting to know the hemolysis data in these patients, since we speculate that patients treated with $\mathrm{DO}_{2}$-guided perfusion, with lower CPB flows, may show better hemolysis compared with controls. ${ }^{4}$

However, many questions are still open: Operative mortality was greater in the treatment arm, but the number of deaths was small, and this difference did not meet significance. Is this because the trial is underpowered to identify a difference in safety endpoints such as mortality? Was the study sample size adequate? Even though the trial was stopped before the planned number of patients was recruited? How clinically appropriate was the primary endpoint? Finally, a study performed in patients at risk for renal failure that is adequately designed to detect a significant difference in mortality and adverse events is needed.

Giuseppe Santarpino, $M D^{a, b}$ Pasquale Di Molfetta, MD

Marcello Melone, $M D^{c}$

${ }^{a}$ Department of Cardiac Surgery

Paracelsus Medical University

Nuremberg, Germany

${ }^{b}$ Department of Cardiac Surgery

${ }^{c}$ Department of Anaesthesiology

Città di Lecce Hospital_GVM Care \& Research

Lecce, Italy

\title{
References
}

1. Ranucci M, Johnson I, Willcox T, Baker RA, Boer C, Baumann A, et al. Goal-directed perfusion to reduce acute kidney injury: a randomized trial. J Thorac Cardiovasc Surg. 2018;156:1918-27.e2.

2. Perrotti A, Miltgen G, Chevet-Noel A, Durst C, Vernerey D, Bardonnet K, et al. Neutrophil gelatinase-associated lipocalin as early predictor of acute kidney injury after cardiac surgery in adults with chronic kidney failure. Ann Thorac Surg. 2015; 99:864-9.

3. Ranucci M, Frigiola A, Menicanti L, Castelvecchio S, de Vincentis C, Pistuddi V. Aortic cross-clamp time, new prostheses, and outcome in aortic valve replacement. J Heart Valve Dis. 2012;21:732-9.

4. Arensdorf J, Petitt H, Holt D. Improving hemolysis levels associated with cardiotomy suction. Perfusion. 2018;33:612-7. 